\title{
Effect of integrated nutrient management on growth, productivity, quality and nutrient uptake of irrigated yellow sarson (Brassica campestris $L$ var. yellow sarson) in older alluvial soil of West Bengal
}

\author{
Amrit Raj" and R. B Mallick \\ Department of Agronomy, Institute of Agricultural Science, University of Calcutta, 51/2 Hazra Road, Kolkata - \\ 700019 (West Bengal), INDIA \\ "Corresponding author: E-mail: amritagnm@rediffmail.com
}

Received: August 10, 2016; Revised received: February 23, 2017; Accepted: July 11, 2017

\begin{abstract}
A field experiment was conducted during rabi (winter) seasons of 2007-08 and 2008-09 to study the effect of integrated nutrient management on growth, yield, oil content and nutrient uptake of yellow sarson (Brassica campestris $L$ var. yellow sarson) in older alluvial soil of West Bengal. Significantly higher leaf area index (1.75 at 40 days after sowing; DAS), dry matter accumulation $\left(1366.9 \mathrm{~g} / \mathrm{m}^{2}\right.$ at $\left.80 \mathrm{DAS}\right)$ and highest number of siliquae/plant $(118.3)$, number of seeds/siliqua (21.8), seed yield (1.90 t/ha), stover yield (3.86 t/ha) were recorded significantly (at $5 \%$ level) higher with poultry manure (PM) @2.5t/ha +50\%RDF (Recommended Dose of Fertilizer i.e. 80-40-40 of N-P $\mathrm{P}_{2} \mathrm{O}_{5-}$ $\mathrm{K}_{2} \mathrm{O} \mathrm{kg} / \mathrm{ha}$ ) + PSB (phosphate solubilising bacteria) + AZ (Azotobacter) during both the years and on pooled basis. An average of $30.5 \%$ and $233 \%$ increase in seed yield by this treatment was recorded over sole application of RDF and control respectively. Integrated application of PM $(2.5 \mathrm{t} / \mathrm{ha})+50 \%$ RDF + PSB + AZ recorded highest oil content $(43.16 \%)$ and positive effect on soil fertility status. The highest benefit: cost ratios (2.26 and 2.4 in $2007-' 08$ and $2008-' 09$ respectively) were achieved from the use of 50\% RDF + PM (2.5 t/ha) +PSB+AZ.
\end{abstract}

Keywords: Biofertilizer, Nutrient balance, Poultry manure, Vermicompost ,Yellow sarson

\section{INTRODUCTION}

Oilseed crops have been the backbone of agricultural economy of India. Amongst different oilseed crops raised in West Bengal, rapeseed-mustard occupies the largest area and contributes around $75 \%$ to the edible oil basket of the state. Yellow sarson accounts for $90 \%$ coverage in this rape- mustard group in West Bengal, is a winter oilseed crop having high yield potential and relatively high oil content compared to other forms of rapeseed. Although several factors interfere expression of yield potential is restricted mainly due to improper and imbalanced nutrient supply to the crop. Use of chemical fertilizers has increased considerably to meet the higher nutrient requirements of the present day improved varieties. This creates imbalance in nutrient supply leading to decline in soil fertility, crop productivity and sustainability (John et al., 2004). Use of organic matter to meet the nutrient requirement of crops would be an inevitable practice in years to come, particularly for resource poor farmers. However, supplying entire dose of plant nutrients through good quality manures (e.g. poultry manure, vermicompost, goat manure, farm yard manure etc.) are not feasible because their requirement will be several time higher than that of chemical fertilizers because manures content lower amount of plant nutrients. These sources can reduce the mining of soil nutrient and improve soil organic matter, humus and overall soil productivity (Jenssen, 1993). Organic constituents in the humic substances also act as plant growth stimulants (Jenssen, 1993; Palm et al., 1993). A judicious use of organic manures and biofertilizers may be effective not only in sustaining crop productivity and in soil health, but also in supplementing chemical fertilizers of the crops. Balanced application of nutrient enriched organic manures, chemical fertilizers (both water soluble spray and soil applied chemical fertilizers), biofertilizers in an integrated way would be a viable option to improve crop productivity, soil fertility and for cost effectiveness (Sahai, 2005). Keeping these aspects of oilseed production we have conducted an experiment on integrated nutrient management in yellow sarson in farmer's field to investigate the crop performance and improvement of soil fertility status through diverse nutrient management practices.

\section{MATERIALS AND METHODS}

A field experiment was conducted during winter season of 2007-'08 and 2008-'09 at farmer's field (blockPingla) in Paschim Medinipur District of West Bengal $\left(22^{\circ} 14 \mathrm{~N}\right.$ latitude and $87^{\circ} 33^{\circ} \mathrm{E}$ longitude at an altitude of about $14.11 \mathrm{~m}$ above mean sea level). During the crop growing period maximum and minimum tempera- 
tures were $36.0{ }^{0} \mathrm{C}$ and $7.4{ }^{0} \mathrm{C}$ respectively. The total rainfall received during crop growing season in the first year was $4.31 \mathrm{~mm}$. The Soil in the experimental area was medium deep loam, low in organic carbon $(0.39 \%)$ with mild acidic soil reaction $(\mathrm{pH}-5.31)$. The available soil nitrogen $(\mathrm{N})$, phosphorus $(\mathrm{P})$ and potassium (K) were $214.8,20.4$ and $220.12 \mathrm{~kg} / \mathrm{ha}$ respectively. The total $\mathrm{N}, \mathrm{P}$ and $\mathrm{K}$ content in poultry manure were $2.7 \%, 2.05 \%$ and $2.1 \%$ respectively. In vermicompost, total $\mathrm{N}, \mathrm{P}$ and $\mathrm{K}$ were $1.45 \%, 1.92 \%$ and $0.6 \%$ respectively. Two- third $(2 / 3 \mathrm{rd})$ dose of nitrogen and potassium, full recommended dose of phosphorus were applied uniformly in each plot as basal. Remaining 1/3rd dose of nitrogen and potassium were applied at 30 days after sowing (DAS). Organic manures (poultry manure or vermicompost) were pre- inoculated with biofertilizer (phosphate solubilizing bacteria and azotobacter, @6 kg/ha each) few days before application and were kept under shed to build up microbial population. Full dose of pre-inoculated poultry manure and vermicompost ( 5 or $2.5 \mathrm{t} / \mathrm{ha}$ ) were applied right at the time of final land preparation, two days before sowing in plots of respective treatments.

Foliar grade of chemical fertilizer, NPK: 20-20-20 @0.625\% (i.e., 6.25g/lit) and cattle urine (@5\%)were sprayed well over the crop canopy once at $50 \%$ flowering with the help of knapsack sprayer in respective treatments. The sowing of winter (i.e., rabi) crop yellow sarson, cultivar 'Benoy' (B-9) was done by line sowing method with the help of seed drill. Spacing between two adjacent rows was $30 \mathrm{~cm}$ and plant to plant spacing in a row was kept at $10 \mathrm{~cm}$ apart. Thus plant population was maintained at the rate of 333333 plants / hectare. Sowing of seeds was done by $25^{\text {th }}$ November during both the year (2007-08 and 2008-09) of experimentation. The preceding crop (kharif season) was rice during both the year.

The experiment comprising of fourteen different treatments of Integrated Nutrient supply viz. $\mathrm{T}_{1}$ :Absolute Control (No NPK); $\mathrm{T}_{2}: 100 \%$ RDF RDF: Recommended dose of fertilizers $\left(\mathrm{N}: \mathrm{P}_{2} \mathrm{O}_{5}: \mathrm{K}_{2} \mathrm{O}=80: 40: 40 \mathrm{~kg} /\right.$ ha) $\} ; \mathrm{T}_{3}: 100 \% \mathrm{RDF}+\mathrm{Zn}$ (zinc foliar spray); $\mathrm{T}_{4}: \mathrm{VC}$ ( i.e.,vermicompost) (5t/ha) + PSB (i.e.,phosphate solubilising bacteria) + AZ (Azotobacter); $\mathrm{T}_{5}: \mathrm{PM}$ (i.e.,poultry manure) $(5 \mathrm{t} / \mathrm{ha})+\mathrm{PSB}+\mathrm{AZ} ; \mathrm{T}_{6}: 50 \%$ $\mathrm{RDF}+\mathrm{VC}(2.5 \mathrm{t} / \mathrm{ha}) ; \mathrm{T}_{7}: 50 \% \mathrm{RDF}+\mathrm{PM}(2.5 \mathrm{t} / \mathrm{ha}) ;$ $\mathrm{T}_{8}: 50 \% \mathrm{RDF}+\mathrm{VC}(2.5 \mathrm{t} / \mathrm{ha})+\mathrm{PSB}+\mathrm{AZ} ; \mathrm{T}_{9}: 50 \%$ $\mathrm{RDF}+\mathrm{PM}(2.5 \mathrm{t} / \mathrm{ha})+\mathrm{PSB}+\mathrm{AZ} ; \mathrm{T}_{10}: 50 \% \mathrm{RDF}+$ VC $(2.5 \mathrm{t} / \mathrm{ha})+1$ Foliar Spray of NPK-20-20-20 (@0.625\%); $\mathrm{T}_{11}: 50 \% \mathrm{RDF}+\mathrm{PM}(2.5 \mathrm{t} / \mathrm{ha})+1$ Foliar Spray of NPK-20-20-20(@0.625\%); T 12 :50\% RDF + VC $(2.5 \mathrm{t} / \mathrm{ha})+1$ Foliar Spray of Cow Urine (5\%); $\mathrm{T}_{13}: 25 \% \mathrm{RDF}+\mathrm{VC}(2.5 \mathrm{t} / \mathrm{ha})+1$ Foliar Spray of NPK -20-20-20 (@0.625\%)+ PSB + AZ; T $14: 25 \%$ RDF + PM (2.5 t/ha) + 1 Foliar Spray of NPK-20-20-20 (@ $0.625 \%)+$ PSB +AZ , were laid out in a random- ized block design with three replications on older alluvial soil of medium land under irrigated condition. The gross plot size was $5 \mathrm{~m}$ x $3 \mathrm{~m}$. The seed yield was calculated on the basis of net plot $(4.5 \mathrm{~m} \mathrm{x} 2.5 \mathrm{~m})$ harvested. Observations like growth attributese.g. plant height (cm), leaf area index (LAI), dry weight $/ \mathrm{m}^{2}$, crop growth rate (CGR, g/m $/ \mathrm{m}^{2}$ day) were taken at 20, 40, 60, 80 day after sowing (DAS) and at harvest. For this purpose, five randomly selected plants from a row of each plot were uprooted, cleaned and observations were recorded and averaged. Plant height was measured by using a meter scale. Dry matter of plants were estimated by weighing above ground parts (all leaves, branches and stems etc.,) after being dried them in a hot air oven (at $65^{\circ} \mathrm{C}$ to $70^{\circ} \mathrm{C}$ ) till the constant weight were obtained. Border rows were not considered for sampling. Yield attributes at the time of harvest were recorded to assess their contribution to yield. Total number of siliquae from five randomly selected plants from each plot were counted, averaged (total siliquae/5) and recorded as siliquae/ plant. The 1000 seeds were counted from the lot of respective treatments, weighed and expressed as 1000 seed weight. The seed and stover yields were computed from net plots (except border rows) and expressed in $\mathrm{kg} / \mathrm{ha}$. The economical parameters, like net monetary returns and benefit:cost ratio were worked out by using the prevailing market price of the inputs and produce in the locality. The oil content was estimated by Pulse Nuclear Magnetic Resonance (NMR) technique (Tiwari and Burk, 1980).

Soil samples were analysed as per procedure described by Jackson (1973). Soil organic carbon and the available $\mathrm{N}$ were evaluated by the Wakely and Black (1963) method and the micro-Kjeldahl digestion method (Bremmer and Mulvaney, 1982) Available P in soil was extracted by the method of Bray and Kurtz (1945), while available $\mathrm{K}$ content was extracted with neutral $1 \mathrm{M}$ NH4OAc at a soil solution ratio of 1:10 and measured by flame photometry.Plant sample analysis for nitrogen content was estimated by wet digestion with concentrated sulphuric acid and distillation process (Jackson, 1973), Plant phosphorus and potassium content was estimated by Tri-acid $\left(\mathrm{HNO}_{3}: \mathrm{HClO}_{4}: \mathrm{H}_{2} \mathrm{SO}_{4}\right)$ digestion process followed by spectrophotometric analysis with vanadomolybdate reagent (Jackson, 1973). Nutrient balance in the soil was worked out on the basis of initial and final values of N, P and $\mathrm{K}$ respectively. The statistical analysis of data was performed using Microsoft Excel and MSTAT-C softwares following the procedure of Gomez and Gomez (1984). Statistical significance between mean differences among treatments for various parameters was analyzed using critical differences (CD) at 0.05 probability level. As the error variance of the two year experiment were found to be homogenous (through Bartlett's test) so the results were pooled. 
Table 1. Effect of integrated nutrient supply on growth attributes of yellow sarson (data pooled of two years,2007-08 and 200809).

\begin{tabular}{lcccc}
\hline Treatments & $\begin{array}{c}\text { Plant } \\
\text { Height }(\mathbf{c m}) \\
\text { (at harvest) }\end{array}$ & $\begin{array}{c}\text { Leaf area } \\
\text { Index } \\
\text { (at 40 DAS) }\end{array}$ & $\begin{array}{c}\text { Dry weight } \\
\left(\mathbf{g} / \mathbf{~ m}^{\mathbf{2}}\right) \\
(\mathbf{a t 8 0} \mathbf{D A S})\end{array}$ & $\begin{array}{c}\text { Crop growth rate } \\
\left(\mathbf{g} / \mathbf{m}^{\mathbf{2}} / \mathbf{d a y}\right) \\
\mathbf{( 4 0 - 6 0 D A S})\end{array}$ \\
\hline T1 & 36.53 & 0.61 & 811.07 & 17.76 \\
T2 & 106.98 & 1.26 & 1287.28 & 25.12 \\
T3 & 106.66 & 1.29 & 1265.67 & 25.22 \\
T4 & 93.96 & 0.91 & 1284.68 & 26.56 \\
T5 & 94.95 & 0.95 & 1285.6 & 26.45 \\
T6 & 107.69 & 1.28 & 1306.92 & 26.72 \\
T7 & 108.01 & 1.75 & 1334.31 & 27.33 \\
T8 & 112.39 & 1.75 & 1352.45 & 27.69 \\
T9 & 113.6 & 1.27 & 1366.92 & 27.84 \\
T10 & 108.79 & 1.32 & 1305.47 & 26.53 \\
T11 & 112.33 & 1.3 & 1342.51 & 27.26 \\
T12 & 1.27 & 1299.07 & 26.45 \\
T13 & 108.84 & 1.3 & 1318.78 & 27.01 \\
T14 & 111.9 & 0.03 & 1332.93 & 27.31 \\
CD $(\mathrm{P}=0.05)$ & 0.55 & 39.64 & 0.41 \\
\hline
\end{tabular}

$\mathrm{T}_{1}$ : Control; $\mathrm{T}_{2}: 100 \% \mathrm{RDF} ; \mathrm{T} 3: 100 \% \mathrm{RDF}+\mathrm{Zn} ; \mathrm{T}_{4}: \mathrm{VC}(5 \mathrm{t} / \mathrm{ha})+\mathrm{PSB}+\mathrm{AZ} ; \mathrm{T}_{5}: \mathrm{PM}(5 \mathrm{t} / \mathrm{ha})+\mathrm{PSB}+\mathrm{AZ} ; \mathrm{T}_{6}: 50 \% \mathrm{RDF}+$ VC (2.5 t/ha); $\mathrm{T}_{7}: 50 \% \mathrm{RDF}+\mathrm{PM}(2.5 \mathrm{t} / \mathrm{ha}) ; \mathrm{T}_{8}: 50 \% \mathrm{RDF}+\mathrm{VC}(2.5 \mathrm{t} / \mathrm{ha})+\mathrm{PSB}+\mathrm{AZ} ; \mathrm{T}_{9}: 50 \% \mathrm{RDF}+\mathrm{PM}(2.5 \mathrm{t} / \mathrm{ha})+\mathrm{PSB}$ + AZ; $\mathrm{T}_{10}: 50 \% \mathrm{RDF}+\mathrm{VC}(2.5 \mathrm{t} / \mathrm{ha})+1$ F.S. of NPK-20-20-20 (@0.625\%); $\mathrm{T}_{11}: 50 \% \mathrm{RDF}+\mathrm{PM}(2.5 \mathrm{t} / \mathrm{ha})+1$ F.S. of NPK20-20-20(@0.625\%); $\mathrm{T}_{12}: 50 \% \mathrm{RDF}+\mathrm{VC}(2.5 \mathrm{t} / \mathrm{ha})+1 \mathrm{~F} . \mathrm{S}$. of Cow Urine $(5 \%) ; \mathrm{T}_{13}: 25 \% \mathrm{RDF}+\mathrm{VC}(2.5 \mathrm{t} / \mathrm{ha})+1 \mathrm{~F} . \mathrm{S}$. of NPK-20-20-20 (@0.625\%)+PSB + AZ; $\mathrm{T}_{14}: 25 \%$ RDF + PM (2.5 t/ha)+1 F.S. of NPK-20-20-20(@0.625\%)+PSB + AZ. (DAS= Days After Sowing). $\left[\mathrm{g} / \mathrm{m}^{2}=\right.$ gram per square meter; DAS= Days After Sowing $]$

Table 2. Effect of integrated nutrient supply on yield components, seed yield, stover yield and seed oil content of yellow sarson \{data pooled of two years, (2007-08 and 2008-09) at harvest\}.

\begin{tabular}{lccccccc}
\hline Treatments & $\begin{array}{c}\text { No.of } \\
\text { branches/ plant }\end{array}$ & $\begin{array}{c}\text { No.of } \\
\text { Siliquae/ plant }\end{array}$ & $\begin{array}{c}\text { No.of seeds/ } \\
\text { siliqua }\end{array}$ & $\begin{array}{c}\text { 1000-seed } \\
\text { weight (g) }\end{array}$ & $\begin{array}{c}\text { seed } \\
\text { yield (t/ha) }\end{array}$ & $\begin{array}{c}\text { Stover } \\
\text { yield (t/ha) }\end{array}$ & $\begin{array}{c}\text { Seed oil con- } \\
\text { tent (\%) }\end{array}$ \\
\hline T1 & 5.16 & 49.51 & 16.27 & 1.53 & 0.57 & 1.05 & 35.67 \\
T2 & 10.55 & 92.4 & 20.59 & 2.11 & 1.32 & 2.68 & 40.59 \\
T3 & 11.04 & 94.45 & 20.21 & 2.16 & 1.35 & 2.78 & 40.28 \\
T4 & 9.95 & 81.55 & 19.58 & 2.09 & 1.04 & 1.79 & 39.32 \\
T5 & 10.24 & 86.67 & 19.49 & 2.15 & 1.12 & 1.97 & 39.27 \\
T6 & 11.45 & 94.78 & 20.69 & 2.26 & 1.48 & 2.65 & 40.55 \\
T7 & 12.09 & 105.9 & 20.94 & 2.15 & 1.6 & 2.93 & 41.75 \\
T8 & 12.83 & 11.23 & 21.68 & 2.17 & 1.72 & 3.15 & 42.67 \\
T9 & 13.89 & 118.31 & 21.81 & 2.29 & 1.9 & 3.86 & 43.16 \\
T10 & 11.97 & 104.19 & 20.05 & 2.32 & 1.46 & 2.64 & 43.08 \\
T11 & 12.16 & 112.19 & 20.82 & 2.25 & 1.69 & 2.92 & 42.88 \\
T12 & 11.13 & 94.75 & 20.56 & 2.22 & 1.43 & 2.45 & 40.95 \\
T13 & 11.39 & 101.07 & 20.83 & 2.19 & 1.52 & 2.79 & 40.24 \\
T14 & 11.9 & 107.35 & 20.82 & 2.23 & 1.63 & 3.07 & 41.06 \\
CD (P=0.05) & 0.36 & 1.62 & 0.67 & 0.15 & 0.04 & 0.08 & 0.67 \\
\hline
\end{tabular}

$\mathrm{T}_{1}$ : Control; $\mathrm{T}_{2}: 100 \% \mathrm{RDF} ; \mathrm{T} 3: 100 \% \mathrm{RDF}+\mathrm{Zn} ; \mathrm{T}_{4}: \mathrm{VC}(5 \mathrm{t} / \mathrm{ha})+\mathrm{PSB}+\mathrm{AZ} ; \mathrm{T}_{5}: \mathrm{PM}(5 \mathrm{t} / \mathrm{ha})+\mathrm{PSB}+\mathrm{AZ} ; \mathrm{T}_{6}: 50 \% \mathrm{RDF}+$ $\mathrm{VC}(2.5 \mathrm{t} / \mathrm{ha}) ; \mathrm{T}_{7}: 50 \% \mathrm{RDF}+\mathrm{PM}(2.5 \mathrm{t} / \mathrm{ha}) ; \mathrm{T}_{8}: 50 \% \mathrm{RDF}+\mathrm{VC}(2.5 \mathrm{t} / \mathrm{ha})+\mathrm{PSB}+\mathrm{AZ} ; \mathrm{T}_{9}: 50 \% \mathrm{RDF}+\mathrm{PM}(2.5 \mathrm{t} / \mathrm{ha})+\mathrm{PSB}$ + AZ; $\mathrm{T}_{10}: 50 \%$ RDF + VC (2.5 t/ha) + 1 F.S. of NPK-20-20-20 (@0.625\%); T $11: 50 \%$ RDF + PM (2.5 t/ha) + 1 F.S. of NPK20-20-20(@0.625\%); $\mathrm{T}_{12}: 50 \% \mathrm{RDF}+\mathrm{VC}(2.5 \mathrm{t} / \mathrm{ha})+1$ F.S. of Cow Urine (5\%); $\mathrm{T}_{13}: 25 \% \mathrm{RDF}+\mathrm{VC}(2.5 \mathrm{t} / \mathrm{ha})+1 \mathrm{~F} . \mathrm{S}$. of NPK-20-20-20(@0.625\%)+PSB + AZ; $\mathrm{T}_{14}: 25 \%$ RDF + PM (2.5 t/ha)+1 F.S. of NPK-20-20-20(@0.625\%)+PSB + AZ.

\section{RESULTS AND DISCUSSION}

Growth attributes: The growth parameters like plant height $(\mathrm{cm})$, leaf area index, dry matter production, crop growth rate $\left(\mathrm{g} / \mathrm{m}^{2} /\right.$ day) were affected significantly by different combinations of organic and chemical plant nutrient sources on pooled basis (Table 1). The tallest plants were observed by application of $50 \%$
$\mathrm{RDF}+\mathrm{PM}(2.5 \mathrm{t} / \mathrm{ha})+\mathrm{PSB}+\mathrm{AZ}$ followed by $50 \%$ $\mathrm{RDF}+\mathrm{VC}(2.5 \mathrm{t} / \mathrm{ha})+\mathrm{PSB}+\mathrm{AZ}$ which was at par with $25 \%$ RDF + PM $(2.5 \mathrm{t} / \mathrm{ha})+\mathrm{PSB}+\mathrm{AZ}+\mathrm{F} . \mathrm{S}$ of NPK 20-20-20@ 0.625\%. The LAI increased significantly with combined application of poultry manure or vermicompost and $50 \%$ reduced dose of RDF along with biofertilizer and / or foliar application of NPK. The dry weights of different treatments of yellow sar- 
Amrit Raj and R.B Mallick/ J. Appl. \& Nat. Sci. 9 (3): 1411 - 1418 (2017)

Table 3. Effect of integrated nutrient management on soil nitrogen balance after second year harvest of yellow sarson.

\begin{tabular}{lccccc}
\hline Treatment & $\begin{array}{c}\text { Initial available } \\
\text { soil N+added N } \\
\text { To soil (kg/ha), }\end{array}$ & $\begin{array}{c}\text { Total N uptake, } \\
\text { at harvest } \\
\text { by crop } \mathbf{( k g / h a )},\end{array}$ & $\begin{array}{c}\text { Expected } \\
\text { balance } \\
\text { (kg/ha) }\end{array}$ & $\begin{array}{c}\text { Actual } \\
\text { balance } \\
\text { (kg/ha) }\end{array}$ & $\begin{array}{c}\text { Net gain } \\
\text { or loss } \\
\text { (kg/ha) }\end{array}$ \\
\hline T1 & 168.0 & 34.45 & 133.5 & 184.9 & 51.36 \\
T2 & 248.0 & 146.38 & 101.6 & 237.5 & 135.9 \\
T3 & 248.0 & 165.88 & 82.12 & 228.5 & 146.4 \\
T4 & 240.5 & 105.46 & 135.0 & 264.8 & 129.8 \\
T5 & 303.0 & 114.76 & 188.2 & 266.7 & 78.46 \\
T6 & 244.3 & 168.95 & 75.30 & 289.6 & 214.3 \\
T7 & 275.5 & 192.94 & 82.56 & 301.0 & 218.4 \\
T8 & 244.3 & 223.22 & 21.03 & 303.0 & 282.0 \\
T9 & 275.5 & 169.32 & 23.00 & 298.0 & 275.0 \\
T10 & 244.3 & 189.06 & 74.93 & 265.1 & 190.2 \\
T11 & 148.7 & 86.44 & 291.8 & 205.4 \\
T12 & 275.5 & 180.97 & 95.55 & 260.2 & 164.7 \\
T13 & 244.3 & 201.48 & 43.28 & 305.5 & 262.2 \\
T14 & 224.3 & 54.02 & 296 & 242 \\
\hline
\end{tabular}

Initial value: $\{$ available soil $\mathrm{N}$, (second year) $\}=168 \mathrm{~kg} / \mathrm{ha}$

$\mathrm{T}_{1}:$ Control; $\mathrm{T}_{2}: 100 \% \mathrm{RDF} ; \mathrm{T} 3: 100 \% \mathrm{RDF}+\mathrm{Zn} ; \mathrm{T}_{4}: \mathrm{VC}(5 \mathrm{t} / \mathrm{ha})+\mathrm{PSB}+\mathrm{AZ} ; \mathrm{T}_{5}: \mathrm{PM}(5 \mathrm{t} / \mathrm{ha})+\mathrm{PSB}+\mathrm{AZ} ; \mathrm{T}_{6}: 50 \% \mathrm{RDF}+$ $\mathrm{VC}(2.5 \mathrm{t} / \mathrm{ha}) ; \mathrm{T}_{7}: 50 \% \mathrm{RDF}+\mathrm{PM}(2.5 \mathrm{t} / \mathrm{ha}) ; \mathrm{T}_{8}: 50 \% \mathrm{RDF}+\mathrm{VC}(2.5 \mathrm{t} / \mathrm{ha})+\mathrm{PSB}+\mathrm{AZ} ; \mathrm{T}_{9}: 50 \% \mathrm{RDF}+\mathrm{PM}(2.5 \mathrm{t} / \mathrm{ha})+\mathrm{PSB}$ + AZ; $\mathrm{T}_{10}: 50 \%$ RDF + VC (2.5 t/ha) + 1 F.S. of NPK-20-20-20 (@0.625\%); $\mathrm{T}_{11}: 50 \% \mathrm{RDF}+\mathrm{PM}(2.5 \mathrm{t} / \mathrm{ha})+1 \mathrm{~F} . \mathrm{S}$. of NPK20-20-20(@0.625\%); $\mathrm{T}_{12}: 50 \% \mathrm{RDF}+\mathrm{VC}(2.5 \mathrm{t} / \mathrm{ha})+1 \mathrm{~F} . \mathrm{S}$. of Cow Urine (5\%); $\mathrm{T}_{13}: 25 \% \mathrm{RDF}+\mathrm{VC}(2.5 \mathrm{t} / \mathrm{ha})+1 \mathrm{~F} . \mathrm{S}$. of NPK-20-20-20 (@0.625\%)+PSB + AZ; T $14: 25 \%$ RDF + PM (2.5 t/ha)+1 F.S. of NPK-20-20-20 (@0.625\%)+ PSB + AZ.

Table 4. Effect of integrated nutrient management on soil phosphorus balance after second year harvest of yellow sarson.

\begin{tabular}{|c|c|c|c|c|c|}
\hline Treatment & $\begin{array}{c}\text { Initial available Soil } \\
\mathrm{P}_{2} \mathrm{O}_{5}+\text { added } \mathrm{P} \text { to soil } \\
(\mathrm{kg} / \mathrm{ha})\end{array}$ & $\begin{array}{c}\text { Total } \\
\mathrm{P}_{2} \mathrm{O}_{5} \text { uptake, } \\
\text { at harvest by crop }(\mathrm{kg} / \mathrm{ha})\end{array}$ & $\begin{array}{l}\text { Expected } \\
\text { balance } \\
(\mathrm{kg} / \mathrm{ha})\end{array}$ & $\begin{array}{c}\text { Actual } \\
\text { balance } \\
\text { (kg/ha) }\end{array}$ & $\begin{array}{c}\text { Net gain } \\
\text { Or loss }(\mathrm{kg} / \mathrm{ha})\end{array}$ \\
\hline$\overline{\mathrm{T} 1}$ & 14.8 & 6.7138 & 8.086 & 13.4 & 5.314 \\
\hline $\mathrm{T} 2$ & 54.8 & 31.714 & 23.09 & 18.9 & -4.186 \\
\hline $\mathrm{T} 3$ & 54.8 & 33.645 & 21.15 & 20.5 & -0.655 \\
\hline $\mathrm{T} 4$ & 110.8 & 25.481 & 85.32 & 27.5 & -57.82 \\
\hline $\mathrm{T} 5$ & 117.3 & 28.235 & 89.07 & 28.6 & -60.47 \\
\hline T6 & 82.8 & 36.458 & 46.34 & 29.9 & -16.44 \\
\hline $\mathrm{T} 7$ & 86.05 & 42.731 & 43.32 & 44.9 & 1.581 \\
\hline T8 & 82.8 & 55.512 & 27.29 & 41.8 & 14.51 \\
\hline T9 & 86.05 & 52.875 & 33.18 & 47 & 13.83 \\
\hline $\mathrm{T} 10$ & 82.8 & 38.243 & 44.56 & 39.8 & -4.757 \\
\hline $\mathrm{T} 11$ & 86.05 & 47.163 & 38.89 & 43.8 & 4.913 \\
\hline $\mathrm{T} 12$ & 82.8 & 35.836 & 46.96 & 38.9 & -8.064 \\
\hline $\mathrm{T} 13$ & 72.8 & 44.291 & 28.51 & 45.8 & 17.29 \\
\hline $\mathrm{T} 14$ & 76.05 & 52.078 & 23.97 & 42.7 & 18.73 \\
\hline
\end{tabular}

Initial value: $\left\{\right.$ available soil $\mathrm{P}_{2} \mathrm{O}_{5}$, (second year) $\}=14.8 \mathrm{~kg} / \mathrm{ha}$

$\mathrm{T}_{1}:$ Control; $\mathrm{T}_{2}: 100 \% \mathrm{RDF} ; \mathrm{T} 3: 100 \% \mathrm{RDF}+\mathrm{Zn} ; \mathrm{T}_{4}: \mathrm{VC}(5 \mathrm{t} / \mathrm{ha})+\mathrm{PSB}+\mathrm{AZ} ; \mathrm{T}_{5}: \mathrm{PM}(5 \mathrm{t} / \mathrm{ha})+\mathrm{PSB}+\mathrm{AZ} ; \mathrm{T}_{6}: 50 \% \mathrm{RDF}+$ $\mathrm{VC}(2.5 \mathrm{t} / \mathrm{ha}) ; \mathrm{T}_{7}: 50 \% \mathrm{RDF}+\mathrm{PM}(2.5 \mathrm{t} / \mathrm{ha}) ; \mathrm{T}_{8}: 50 \% \mathrm{RDF}+\mathrm{VC}(2.5 \mathrm{t} / \mathrm{ha})+\mathrm{PSB}+\mathrm{AZ} ; \mathrm{T}_{9}: 50 \% \mathrm{RDF}+\mathrm{PM}(2.5 \mathrm{t} / \mathrm{ha})+\mathrm{PSB}$ $+\mathrm{AZ} ; \mathrm{T}_{10}: 50 \% \mathrm{RDF}+\mathrm{VC}(2.5 \mathrm{t} / \mathrm{ha})+1 \mathrm{~F} . \mathrm{S}$. of NPK-20-20-20 (@0.625\%); T $11: 50 \% \mathrm{RDF}+\mathrm{PM}(2.5 \mathrm{t} / \mathrm{ha})+1 \mathrm{~F} . \mathrm{S}$. of NPK20-20-20(@0.625\%); $\mathrm{T}_{12}: 50 \% \mathrm{RDF}+\mathrm{VC}(2.5 \mathrm{t} / \mathrm{ha})+1 \mathrm{~F} . \mathrm{S}$. of Cow Urine (5\%); $\mathrm{T}_{13}: 25 \% \mathrm{RDF}+\mathrm{VC}(2.5 \mathrm{t} / \mathrm{ha})+1 \mathrm{~F} . \mathrm{S} . \mathrm{of}$ NPK-20-20-20 (@0.625\%)+PSB + AZ; T $14: 25 \%$ RDF + PM (2.5 t/ha)+1 F.S. of NPK-20-20-20 (@0.625\%)+ PSB + AZ.

son crop differed significantly. Integration of different nutritional sources recorded higher dry weights than sole use of chemical fertilizer or sole organic manure (VC / PM) at later growth stages. Significantly higher total dry matter production was recorded at different growth stages of yellow sarson crop in T9 AZ + PSB + PM $(2.5 \mathrm{t} / \mathrm{ha})+50 \% \mathrm{RDF}\}$ which was closely followed by T8 $\{\mathrm{AZ}+\mathrm{PSB}+\mathrm{VC}(2.5 \mathrm{t} / \mathrm{ha})+50 \% \mathrm{RDF}\}$ compared to others. Treatments clearly indicated the beneficial effects of Azotobacter, PSB in presence of poultry manure and vermicompost. Increased dry matter in these treatments might be due to positive role of the biofertilizer in presence of organic manures. Supply of the required nutrients through organic and inorganic sources and biofertilizer facilitated balanced nutrient of the crop, which resulted in enhanced dry 
Amrit Raj and R.B Mallick/ J. Appl. \& Nat. Sci. 9 (3): 1411 - 1418 (2017)

Table 5. Effect of integrated nutrient management on soil potassium balance after second year harvest of yellow sarson.

\begin{tabular}{|c|c|c|c|c|c|}
\hline Treatment & $\begin{array}{c}\text { Initial available soil } \\
\mathrm{K}_{2} \mathrm{O}+\text { added } \mathrm{K}_{2} \mathrm{O} \text { to } \\
\text { soil }(\mathrm{kg} / \mathrm{ha})\end{array}$ & $\begin{array}{c}\text { Total } \mathrm{K}_{2} \mathrm{O} \text { uptake, } \\
\text { at harvest by crop }(\mathrm{kg} / \mathrm{ha})\end{array}$ & $\begin{array}{c}\text { Expected balance } \\
\text { (kg/ha) }\end{array}$ & $\begin{array}{c}\text { Actual Balance } \\
(\mathrm{kg} / \mathrm{ha})\end{array}$ & $\begin{array}{l}\text { Net gain or } \\
\text { loss (kg/ha) }\end{array}$ \\
\hline$\overline{\mathrm{T} 1}$ & 213 & 12.87 & 200 & 200 & -0.14 \\
\hline T2 & 253 & 136.7 & 116 & 271 & 154.6 \\
\hline T3 & 253 & 145.7 & 107 & 267 & 159.8 \\
\hline $\mathrm{T} 4$ & 243 & 91.22 & 151 & 226 & 74.21 \\
\hline T5 & 318 & 104.3 & 213 & 231 & 17.49 \\
\hline T6 & 248 & 150.6 & 97.1 & 322 & 225.2 \\
\hline $\mathrm{T} 7$ & 285 & 171.5 & 114 & 325 & 211.4 \\
\hline T8 & 248 & 209.6 & 38.1 & 327 & 289.1 \\
\hline T9 & 285 & 283.7 & 1.46 & 340 & 338.9 \\
\hline $\mathrm{T} 10$ & 248 & 153.7 & 94 & 263 & 168.8 \\
\hline T11 & 285 & 177.4 & 108 & 276 & 168.4 \\
\hline $\mathrm{T} 12$ & 248 & 160 & 87.7 & 335 & 247.5 \\
\hline $\mathrm{T} 13$ & 238 & 166.9 & 70.8 & 265 & 193.7 \\
\hline $\mathrm{T} 14$ & 275 & 187.5 & 87.7 & 273 & 185 \\
\hline
\end{tabular}

Initial value: $\left\{\right.$ available soil $\mathrm{K}_{2} \mathrm{O}$, (second year) $\}=201.01 \mathrm{~kg} / \mathrm{ha}$

$\mathrm{T}_{1}$ : Control; $\mathrm{T}_{2}: 100 \% \mathrm{RDF} ; \mathrm{T} 3: 100 \% \mathrm{RDF}+\mathrm{Zn} ; \mathrm{T}_{4}: \mathrm{VC}(5 \mathrm{t} / \mathrm{ha})+\mathrm{PSB}+\mathrm{AZ} ; \mathrm{T}_{5}: \mathrm{PM}(5 \mathrm{t} / \mathrm{ha})+\mathrm{PSB}+\mathrm{AZ} ; \mathrm{T}_{6}: 50 \% \mathrm{RDF}+$ $\mathrm{VC}(2.5 \mathrm{t} / \mathrm{ha}) ; \mathrm{T}_{7}: 50 \% \mathrm{RDF}+\mathrm{PM}(2.5 \mathrm{t} / \mathrm{ha}) ; \mathrm{T}_{8}: 50 \% \mathrm{RDF}+\mathrm{VC}(2.5 \mathrm{t} / \mathrm{ha})+\mathrm{PSB}+\mathrm{AZ} ; \mathrm{T}_{9}: 50 \% \mathrm{RDF}+\mathrm{PM}(2.5 \mathrm{t} / \mathrm{ha})+\mathrm{PSB}$ + AZ; $\mathrm{T}_{10}: 50 \%$ RDF + VC (2.5 t/ha) + 1 F.S. of NPK-20-20-20 (@0.625\%); T $11: 50 \%$ RDF + PM (2.5 t/ha) + 1 F.S. of NPK20-20-20(@0.625\%); $\mathrm{T}_{12}: 50 \% \mathrm{RDF}+\mathrm{VC}(2.5 \mathrm{t} / \mathrm{ha})+1 \mathrm{~F} . \mathrm{S}$. of Cow Urine (5\%); $\mathrm{T}_{13}: 25 \% \mathrm{RDF}+\mathrm{VC}(2.5 \mathrm{t} / \mathrm{ha})+1 \mathrm{~F} . \mathrm{S}$. of NPK-20-20-20(@0.625\%)+PSB+AZ; T $14: 25 \%$ RDF + PM (2.5 t/ha)+1 F.S. of NPK-20-20-20(@0.625\%)+PSB+AZ.

Table 6. Economics of integrated nutrient supply of yellow sarson (two years).

\begin{tabular}{|c|c|c|c|c|c|c|}
\hline \multirow[t]{2}{*}{ Treatments } & \multicolumn{2}{|c|}{ Gross return (Rs/ha) } & \multicolumn{2}{|c|}{ Net return (Rs/ha) } & \multicolumn{2}{|c|}{ Benefit:Cost ratio } \\
\hline & $2007-08$ & 2008-09 & $2007-08$ & 2008-09 & $2007-08$ & 2008-09 \\
\hline$\overline{\mathrm{T} 1}$ & 25600 & 22440 & 10738.3 & 7572.8 & 0.72 & 0.5 \\
\hline $\mathrm{T} 2$ & 54800 & 55880 & 37505.3 & 38576.85 & 2.16 & 2.22 \\
\hline T3 & 56000 & 57200 & 37794.5 & 38986.05 & 2.07 & 2.13 \\
\hline $\mathrm{T} 4$ & 43200 & 44440 & 8088.3 & 9319.85 & 0.22 & 0.26 \\
\hline T5 & 46400 & 47520 & 16288.3 & 17399.85 & 0.53 & 0.57 \\
\hline T6 & 61200 & 62920 & 34871.8 & 36583.35 & 1.32 & 1.38 \\
\hline $\mathrm{T} 7$ & 66000 & 68200 & 42171.8 & 44363.35 & 1.76 & 1.86 \\
\hline $\mathrm{T} 8$ & 70800 & 73480 & 44421.8 & 47093.35 & 1.68 & 1.78 \\
\hline T9 & 78000 & 81400 & 54121.8 & 57513.35 & 2.26 & 2.4 \\
\hline $\mathrm{T} 10$ & 61600 & 61160 & 34921.8 & 34473.35 & 1.3 & 1.28 \\
\hline T11 & 70800 & 71280 & 46621.8 & 47093.35 & 1.92 & 1.94 \\
\hline T12 & 60400 & 59840 & 33856.8 & 33288.35 & 1.27 & 1.24 \\
\hline T13 & 62800 & 64680 & 36655.05 & 38526.6 & 1.4 & 1.46 \\
\hline T14 & 67200 & 69520 & 43555.05 & 45866.6 & 1.83 & 1.93 \\
\hline
\end{tabular}

$\mathrm{T}_{1}:$ Control; $\mathrm{T}_{2}: 100 \% \mathrm{RDF} ; \mathrm{T} 3: 100 \% \mathrm{RDF}+\mathrm{Zn} ; \mathrm{T}_{4}: \mathrm{VC}(5 \mathrm{t} / \mathrm{ha})+\mathrm{PSB}+\mathrm{AZ} ; \mathrm{T}_{5}: \mathrm{PM}(5 \mathrm{t} / \mathrm{ha})+\mathrm{PSB}+\mathrm{AZ} ; \mathrm{T}_{6}: 50 \% \mathrm{RDF}+$ $\mathrm{VC}(2.5 \mathrm{t} / \mathrm{ha}) ; \mathrm{T}_{7}: 50 \% \mathrm{RDF}+\mathrm{PM}(2.5 \mathrm{t} / \mathrm{ha}) ; \mathrm{T}_{8}: 50 \% \mathrm{RDF}+\mathrm{VC}(2.5 \mathrm{t} / \mathrm{ha})+\mathrm{PSB}+\mathrm{AZ} ; \mathrm{T}_{9}: 50 \% \mathrm{RDF}+\mathrm{PM}(2.5 \mathrm{t} / \mathrm{ha})+\mathrm{PSB}$ + AZ; $\mathrm{T}_{10}: 50 \%$ RDF + VC (2.5 t/ha)+1 F.S. of NPK-20-20-20 (@0.625\%); T $11: 50 \%$ RDF + PM (2.5 t/ha) + 1 F.S. of NPK20-20-20(@0.625\%); $\mathrm{T}_{12}: 50 \% \mathrm{RDF}+\mathrm{VC}(2.5 \mathrm{t} / \mathrm{ha})+1 \mathrm{~F} . \mathrm{S}$. of Cow Urine (5\%); $\mathrm{T}_{13}: 25 \% \mathrm{RDF}+\mathrm{VC}(2.5 \mathrm{t} / \mathrm{ha})+1 \mathrm{~F} . \mathrm{S}$. of NPK-20-20-20(@0.625\%)+PSB + AZ; $\mathrm{T}_{14}: 25 \%$ RDF + PM (2.5 t/ha)+1 F.S. of NPK-20-20-20(@0.625\%)+ PSB + AZ.

matter production in this crop. Similarly, Maheshbabu et al., (2008) reported the combine use of farm yard manure (FYM) and NPK enhanced growths parameters (like; plant height, LAI etc.) in soybean. Patra et al., (2013) also reported that biofertilizers helped in increasing plant height and leaf chlorophyll content of sunflower.

Yield components: The results on yield components of rapeseed var yellow sarson (Table 2) revealed that application of different treatments of INM exerted profound influence on important yield contributing char- acters like number of branches per plant, number of siliquae per plant, number of seeds per siliqua and 1000 -seed weight $(\mathrm{g})$. Highest number of branches per plant (13.89), number of siliquae/plant (118.3), seeds/ siliqua (21.81) and second highest seed test weight $(2.29 \mathrm{~g})$ were recorded in 50\% RDF + PM $(2.5 \mathrm{t} / \mathrm{ha})+$ PSB + AZ. Positive role of poultry manure in improving yield components of yellow sarson might be due to mineralization of major and minor plant nutrients at slow pace thus increased their availability throughout the growing period of the crop. Behera (2006) also 
reported similar effects of poultry manure on wheat crop. The second highest yield attributes were observed in 50\% RDF + VC (2.5 t/ha) + PSB + AZ treated plots. Mookherjee et al. (2014) also found improvement of yield components and seed yield of yellow sarson by integrated use of vermicompost and chemical fertilizers. Application of PM (2.5 t/ha) / VC (2.5 t/ ha) + biofertilizers (PSB + Azotobacter) along with $50 \% \mathrm{RDF}$ pronouncedly improved number of branches per plant in yellow sarson. Improvement in yield attributes might be due to possible role of Azotobacter through atmospheric nitrogen fixation, better root proliferation, uptake of nutrients and water. Similarly Gudadhe et al. (2005) agreed beneficial role of biofertilizer in improving yield components of Indian mustard.

Seed yield, stover yield and Seed oil content: Seed yield of yellow sarson was markedly influenced as a result of application of plant nutrients from different compatible sources through integrated nutrient management schedule (Table 2). The application of $50 \%$ $\mathrm{RDF}+\mathrm{PM}(2.5 \mathrm{t} / \mathrm{ha})+\mathrm{PSB}+\mathrm{AZ}$ produced significantly (at 5\% level) and appreciably higher seed yield (1.9 t/ ha) over others as well as control indicating that poultry manure mineralized rapidly and provided optimum nutrients to the crop from beginning to end of the crop growth. Poultry manure contains significant amount of nitrogen in both inorganic and organic forms were readily available for plant uptake (Kara et al., 2006). The enhancement of growth and yield by poultry manure along with chemical fertilizers and bio-fertilizers could be due to release of phosphorus and different essential plant nutrients (Djokoto and Stephen, 1961) which enhanced high photosynthetic activities leading to vigorous vegetative and reproductive growth (John et al., 2004). Higher stover yield (3.86 t/ha) was also recorded in 50\% RDF + PM $(2.5 \mathrm{t} / \mathrm{ha})+\mathrm{PSB}+\mathrm{AZ}$ treatment.Application of 50\% RDF + PM (2.5 t/ha) + $\mathrm{PSB}+\mathrm{AZ}$ established its superiority in improving the plant growth, leaf-area index, dry matter production as compared to others. This might be attributed to more photosynthesizing leaf area and also conjectured to its promising macro-and -micro-nutrients contents, their early release solublized the applied and native P (Roy, 1986) and increased absorption (Reddy and Reddy, 1998) which in turn resulted in higher stover yield.

Significantly higher oil content $(43.16 \%)$ was observed with the application of $50 \% \mathrm{RDF}+\mathrm{PM}(2.5 \mathrm{t} / \mathrm{ha})+\mathrm{PSB}+$ AZ. Higher oil content in seeds of yellow sarson under $50 \% \mathrm{RDF}+\mathrm{PM}(2.5 \mathrm{t} / \mathrm{ha})+\mathrm{PSB}+\mathrm{AZ}$ might be due to certain P-containing enzymes in fatty acid synthesis in seeds. The impact of the treatment which added more phosphorus might play a role in enhancing the glucoside content in seed which upon hydrolysis and esterification resulted in higher oil content in seed (Krishnamurthy and Mathan, 1996; Aulakh and Pasaricha, 1988). The essential elements like secondary and micronutrient contained in poultry manure $(\mathrm{PM})$ probably promoted the synthesis of oils. Oil content decreased appreciably with the increasing level of $100 \%$ RDF. The oil content decreased with increasing fertility level could be due to increasing availability of $\mathrm{N}$ which increases the proportion of protein substances in seed and hence oil content was low (Padma et al., 2001).

Nutrient uptake, balance of yellow sarson production: The trend of nutrient uptake by rapeseed varied significantly and appreciably due to different treatments (Tables3-5). The NPK uptake differed significantly due to different treatments. Restricting the application of chemical fertilizers up to $50 \%$ of the recommended dose with integration of poultry manure and supplementary inoculation of AZ and PSB resulted in general higher uptake of N, P and K. This might be attributed to release of phosphorous from insoluble phosphate and fixation of atmospheric nitrogen by Azotobacter in soils. Microorganisms with higher phosphate solubilizing potential increase the availability of soluble phosphate and enhance the plant growth and yield due to better root growth and increases uptake of nutrients (Ponmurugan and Gopi, 2006) which in turn, resulted in physiological changes of the plants on exposure to action of the inoculated microorganisms. In addition, humic acid and micronutrients of poultry manure /vermicompost had greater influence in stimulating roots and regulating metabolism, speeding up the developmental process of plant which, in turn, resulted in better NPK content in yellow sarson plants. Roots also interact extensively with soil microorganism, which further impact plant nutrition either directly by influencing nutrient availability and uptake or indirectly through plant (root) growth promotion. Thus observations are in agreement with those of Singh and Yadav (2008). Integration of organic manure and $\mathrm{AZ}+\mathrm{PSB}$ with 50\% RDF upon decomposition might have improved nutrient availability in soil and thus benefited the crop.

Highest total uptake of N, P and K was observed with $50 \% \mathrm{RDF}+\mathrm{PM}(2.5 \mathrm{t} / \mathrm{ha})+\mathrm{PSB}+\mathrm{AZ}$ than sole application of recommended chemical fertilizer indicating the benefits from the integrated use of fertilizers, manures and bio-inoculants which was also reflected in yield. Higher uptake at 50\% RDF + PM (2.5 t/ha) + $\mathrm{AZ}$ and inoculation with PSB might be due to more nutrients from soil. Similar results were reported by Prasad et al.(2005). The crop was also benefited by some plant growth promoting substances (viz., IAA and gibberellins ) released by PSB and Azotobacter and enrich soil nutrients in available form (Bais et al., 2006 ; Bareae et al., 1976 and Bisht et al., 2009) which lead to higher nutrients uptake. The lowest uptake of $\mathrm{N}, \mathrm{P}$ and $\mathrm{K}$ was in treatments where $\mathrm{N}, \mathrm{P}$ and $\mathrm{K}$ were omitted (control treatment).

The maximum positive balance of $\mathrm{N}$ and $\mathrm{P}$ was recorded with $25 \%$ RDF + VC $(2.5$ t/ha $)+1$ F.S. of NPK-20- 
20-20 (@0.625\%)+ PSB + AZ. While highest K balance was observed in 50\% RDF + PM $(2.5 \mathrm{t} / \mathrm{ha})+\mathrm{PSB}$ + AZ treated plots. Foliar NPK fertilizer increases the availability of plant nutrient to crop and PSB and $\mathrm{Azo}$ tobacter improved the supply of phosphorus (P) and nitrogen $(\mathrm{N})$ to roots, thus maintaining the $\mathrm{N}$ and $\mathrm{P}$ balance in soil.

Economics: In this experiment, the benefit: cost ratio was worked out for different levels of NPK fertilizers, organic manures and biofertilizers (Table - 6). The cost of nutrient treatments was marginally higher with the application of PM/VC $(2.5 \mathrm{t} / \mathrm{ha})+50 \% \mathrm{RDF}+$ Azotobacter + PSB compared to RDF. The net monetary returns and benefit: cost ratio was appreciably higher with 50\% RDF integrated with poultry manure (PM @ 2.5t/ha), Azotobacter and PSB. The superiority of the treatment clearly indicated that the reduced level of chemical fertilizers in conjunction with organic manure and bio-inoculants is a good proposition from economical point of view. This can be attributed to lower input and application cost of biofertilizer and comparatively higher benefit in yield. The fertilizer applied to yellow sarson recorded the highest benefit: cost ratio with 50\% RDF in conjunction with PM (2.5t/ ha), Azotobacter and PSB. This can be attributed to the lower input cost due to reduced level of $50 \% \mathrm{RDF}$ and poultry manure but higher seed yield than that of $100 \%$ RDF. Singh and Agarwal, (2004) and Mookherjee et al. (2014) also reported use of organic manure (like, farm yard manure) in combination with biofertilizers and reduced dose of chemical fertilizers found to increase net return in yellow sarson and wheat respectively .

\section{Conclusion}

The above experiment concluded that application of $50 \%$ RDF (RDF, N: $\mathrm{P}_{2} \mathrm{O}_{5}: \mathrm{K}_{2} \mathrm{O}=80: 40: 40 \mathrm{~kg} / \mathrm{ha}$ ) along with PM (2.5 t/ha) and biofertilizers( PSB + AZ ) have resulted remarkably higher plant height (113.6 $\mathrm{cm}$ ), C.G.R(27.84 g/ $/ \mathrm{m}^{2} /$ day between 40-60 DAS), siliquae/plant (118.31), seeds/siliqua (21.81), seed yield (1.9 t/ha) and seed oil content $(43.16 \%)$ of yellow sarson over other treatments. This treatment has yielded higher soil nutrient balance $(298 \mathrm{kgN} / \mathrm{ha}, 47 \mathrm{~kg}$ $\mathrm{P}_{2} \mathrm{O}_{5} /$ ha and $340 \mathrm{~kg} \mathrm{~K} 2 \mathrm{O} / \mathrm{ha}$ ) over $100 \% \mathrm{RDF}$. Therefor, this combination of nutrient sources can be better nutrient management option of yellow sarson in older alluvial soil of West Bengal.

\section{REFERENCES}

Aulakh, M.S. and Pasaricha, N. S. (1988). Sulphur Fertilization of Oilseeds for Yield and Quality. Proceedings of TSI-symposium on Sulphur in Indian Agriculture held during 9-11 March atNew Delhi.

Bais, H.P., Weir, T. L., Perry, L. G., Gilroy, S. and Vivanco, J. M. (2006). The role of root exudates in rhizosphere interactions with plants and organisms. Annual Review of plant Biology 57: 233-266.
Bareae, J. M., Navorvo, E. and Montonya, E. (1976). Production of plant growth regulators by rhizosphere phosphate solubilizing bacteria. Journal of Applied Bacteriology 40: 129-134.

Behera, U. (2006). Integrated nutrient management for sustaining productivity, quality and profitability of wheatsoybean cropping system. Indian FarmingDec: 32-38.

Bisht, R., Chaturvedi, S., Srivastava, R., Sharma, A.K. and Johri, B.N. (2009). Effect of arbuscular mycorrhizal fungi, Psudomonas fluoresccens and Rhizobium leguminosarum on the growth and nutrient status of Dalbergia sissoo Roxb. Tropical Ecology 50(2): 231-242.

Bray, R.H. and Kurtz, L.T. (1945). Determination of total, organic and available form of phosphorus in soils. Soil Science, 59: 39-45.

Bremmer, J.M and Mulvaney, C.S. (1982). Nutrient total In:Methods of Soil Analysis 2nd ed. Page,A.L. et al., (Eds) pp 595-624. ASA, SSA Madison Wisconsin.

Djokoto, R.K. and Stephens, D. (1961). Thirty long-term fertility trials under continuous cropping in Ghana and crop yield responses to fertilizers and manure. Emperor Journal of Experimental Agriculture 29:181-190.

Gomez, K. A. and Gomez, A. A. (1984). Statistical procedures for agricultural research. John Wiley and sons, Inc. London, UK (2nd edn).

Gudadhe, N. N., Mankar, P.S., Khawale, V.S. and Dongarkar, K. P. (2005). Effect of biofertilizers on growth and yield of mustard (Brassica juncea L.). Journal of Soils and Crops 15(1): 160-162.

Jackson, M.L. (1973). Soil chemical analysis. Prentice Hall of India Pvt. Ltd., New Delhi. Pp. 45-226.

Jenssen, B.H. (1993). Integrated nutrient management: The use of organic and mineral fertilizer. In: 'The role of plant nutrients for sustainable food Crop production in subSaharan Africa', eds. H. Van Reuler, and W. H. Prins, pp. 89-105. Leidschendam, the Netherlands: VKP.

John , L.W., Jamer, D.B., Samuel ,L. T. and Warner, L.W. (2004). Soil Fertility and Fertilizers: An Introduction to nutrient management. Pearson Education, India.

Kara, E.E., Uygur, V. and Erel, A. (2006). The effects of composted poultry wastes on nitrogen mineralization and biological activity in a silt loam soil. Journal of Applied Sciences. 6(11): 2476-2480.

Krishnamurthy,V.V. and Mathan,K.K.(1996). Influence of Sulphur and magnesium on Growth and yield of Sunflower (Helianthus annus L.) .Indian Journal of Agronomy 41(4): 627-629.

Maheshbabu, H. M., Ravi Hunje, N. K., Patil Biradra and Babalad, H. B. (2008). Effect of organic manures on plant growth, seed yield and quality of soybean. Karnataka J. Agric. Sci.,21( 2) : 219-221.

Mookherjee, S., Malik, G.C., Bandyopadhyay, S. and Mitra, B. (2014). The productivity of Brassica rapa var. yellow sarson as influenced by integrated nutrient management practices and seed priming in eastern Indian subhimalayan plains. SAARC Journal of Agriculture 12(1): 106-116.

Padma, B.K., Jagdeshwri, P. and Bharti, M. 2001. Improvement of seed yield and quality through Nutrient Management in Sunflower (Helianthus annus L.) Journal of Oilseed Research 18(1): 48-50.

Palm, C.A., Myers, R .J.K. and Nandwa, S.M. (1993). Combined use of organic and inorganic nutrient sources for 
soil fertility maintenance and replenishment. (In): Replenishing Soil Fertility in Africa, eds. R. J.

Patra, P., Pati, B.K., Ghosh, G.K., Mura,S.S., Saha,A. (2013). Effect of biofertilizers and sulphur on Growth, yield, and oil content of hybrid sunflower (Helianthus annuus. L) In a typical lateritic soil. 2: 603 doi:10.4172/ scientificreports.603 Page.

Ponmurugan, P. and Gopi, C. (2006). Distribution pattern and screening of phosphate solubilizing bacteria isolated from different food and forage crops. Journal of Agronomy 5:600-604.

Prasad, K., Verma, C.P., Verma,R.N. and Pyare, R.(2005). Effect of FYM, gypsum and fertility levels on nutrient uptake by wheat crop in maize-wheat system. Crop Research 29(1): 28-33.

Reddy, B.G. and Reddy, M.S.(1998). Effect of organic manure and nitrogen levels on soil available nutrient status in maize-soybean cropping system. Journal of the Indian Society of Soil Science 46: 474-476.

Roy, R. N.(1986). FAO, fertilizer programme with special reference to integrated plant nutrition system. Fertilizer in agricultural development. Krishak Bharati Cooperative Limited New Delhi, India. pp 149-169.

Sahai, V.N. (2005). Fundamentals of soil,Kalyani Publishers, New Delhi.

Singh, R. and Agarwal, S.K. (2004). Effect of organic manuring and nitrogen fertilization on productivity, nutrient use efficiency and economics of wheat. India Journal of Agronomy 49(1): 49-52.

Singh, R.S., and Yadav, M.K. (2008). Effect of phosphorus and biofertilizers on growth, yield and nutrient uptake of long duration Pigeonpea under rainfed condition. Journal of food legume 21(1):46-48.

Tiwari, P. N. and Burk, W. (1980). Seed oil determination by pulsed nuclear magnetic resonance without weighing and drying seeds. Journal of American Oil Chemist Society 57: 119- 121.

Wakely, A and Black, A.I (1963). An examination of the different methods for determining soil organic matter and proposed modification of the Chromic Acid Titration method. Soil Science, 24: 65-68. 